\title{
The Effectiveness of Tracheal Decannulation Criteria: A Prospective Study Yasmin Aljedaani ${ }^{1}$, Majed AlMourgi ${ }^{2}$, Fahad Alshehri ${ }^{3}$, Mohamed Hatem ${ }^{2}$ \\ ${ }^{1}$ ENT Department, East Jeddah Hospital, Jeddah, ${ }^{2}$ Department of Surgery, College of Medicine, Taif University, Taif, ${ }^{3}$ Radiology Medical imaging Department, Medical College, Qassim University, Saudi Arabia \\ Corresponding author: Majed AlMourgi, e-mail: mourgi@tu.edu.sa
}

\begin{abstract}
Background: Long-term insertion of the tracheostomy tube may lead patients to have complications such as tracheal stenosis, leakage, sepsis or aspiration.

Aim of the work: Our study aimed at applying standards for decannulation of the tracheostomy tube depending on the expertise from prior studies.

Methods: The study included 102 patients who underwent tracheostomy during the study period.

Results: Surgical tracheostomy was done in operating room in $67 \%$ of patients and in intensive care unit by percutaneous dilatational technique in $33 \%$ of patients. Decannulation was successful in $97.7 \%$ of patients and discharged from the hospital within 24-48 hours. Decannulation failed in $2.3 \%$ of patients.

Conclusion: The decision to perform elective decannulation of the tracheostomy tube can be challenging. However, we have demonstrated that decannulation based on objective criteria is effective in improving clinical judgment and weaning safety, with a low failure rate. We did not encounter any mortality or significant adverse events.
\end{abstract}

Keywords: Tracheostomy, Tracheal decannulation, Criteria.

\section{INTRODUCTION}

Tracheostomy is a surgical procedure in which a surgical airway is created in the cervical trachea to facilitate airway protection, ventilation and secretion removal (1). Tracheostomy may be performed surgically or using a percutaneous approach ${ }^{(1,2)}$. Tracheostomy is usually carried out for patients who have trouble in weaning from mechanical ventilation following polytrauma, and those who are unable to clear excessive secretions following a catastrophic neurological insult ${ }^{(2)}$.It is also performed to bypass an upper airway obstruction ${ }^{(3)}$. A tracheostomy is less commonly required in cases of infectious and neoplastic disease. Performing a tracheostomy facilitates the transfer of patients from intensive care to a step-down unit ${ }^{(4)}$.

Historically, the first known descriptions of tracheostomy are from Egyptian tablets from 3600 BC. ${ }^{(5)}$ Aretaeus (2nd century AD) and Galen (2nd to 3rd century AD) documented tracheostomy performed by the Greek physician Asklepiades around $100 \mathrm{BC}^{(6)}$. Islamic physicians 'contributions to the history of tracheostomy during the medieval era have been recently detailed ${ }^{(7)}$. The first, scientific description of successful tracheostomy was by Antonio Musa Brasavola in 1546, who performed the procedure for relief of airway obstruction resulting from enlarged tonsils ${ }^{(8)}$. From 1546 through to 1833, around 28 successful tracheostomies have been recorded in the literature; nearly all of them were performed for relief of upper airway obstruction ${ }^{(8,9)}$. In the early 20th century, tracheostomy was made much safer, and the technical aspects of the procedure were refined and described in detail by the famous surgeon Chevalier Jackson ${ }^{(9)}$. Subsequently, tracheostomy was performed extensively during the polio epidemic in Denmark ${ }^{(10)}$.

A tracheostomy may only be a short-term requirement for most patients and decannulation should be carried out when it is no longer required, to reduce the incidence of complications ${ }^{(6)}$.

Decannulation or weaning is the process of removal of the tracheostomy tube when it is not required as the patient has recovered from the underlying illness and the indication for its initial insertion no longer exists ${ }^{(11)}$.

Patients may be exposed to the possibility of late complications, including tracheal stenosis, leakage, infection or aspiration as a result of prolonged tracheostomy tube placement (12). Decannulation is a basic step in rehabilitation from a critical illness. It carries positive psychological implications as the ability to speak may enhance patient motivation $^{(13)}$. There is no universal agreement regarding the appropriate criteria for tracheostomy weaning. There are various decannulation practices described in the literature ${ }^{(12-14)}$.

\section{AIM OF THE STUDY}

The aim of our study was to apply tracheostomy tube decannulation criteria based on experience from previous studies that addressed this vital transition.

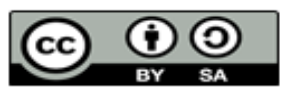

This article is an open access article distributed under the terms and conditions of the Creative Commons Attribution (CC BY-SA) license (http://creativecommons.org/licenses/by/4.0/) 


\section{MATERIAL AND METHODS}

We prospectively included 102 patients who underwent tracheostomy during the study period. The study was carried out in a Tertiary Hospital equipped with trauma and oncology centers, located in the south of Jeddah, in Saudi Arabia from October 2016 to October 2018.

\section{Ethical approval:}

The study was ethically approved by the Institutional Review Board of East Jeddah Hospital and a signed written informed consent was obtained from each patient.

Criteria of decannulation (as shown in table 1):

A protocol-guided approach to decannulation (13-16) was carried out using the following criteria:

1. The patient remained conscious, oriented, and responsive to verbal commands,

2. The patient had recovered from the condition that necessitated performance of a tracheostomy,

3. Procedures that may require endotracheal anesthesia were completed,

4. Patients were no longer ventilator-dependent,

5. Ability to clear secretions with the requirement for tracheal suctioning of no more than once per day,

6. Oxygen saturation maintained at $97 \%$ or more while breathing room air,

7. Although gag reflex was absent in $20 \%$ of the population, the ability to swallow was tested using colored water or by video fluoroscopy as needed,

8. The effectiveness of cough was assessed by the ability to clear tracheal secretions either through the tracheostomy tube or by mouth. This test was performed after deflation of the cuff and occlusion of the proximal end of the tracheostomy tube. A peak flow of at least 160 liters per minute during coughing was used as the cut off for successful decannulation,

9. Flexible fiber optic laryngoscopic examination was performed to ensure airway patency at the glottic and subglottic levels. Rigid bronchoscopic evaluation under general anesthesia was mandatory in the pediatric age group to confirm the absence of any laryngotracheal abnormalities that may complicate decannulation, and

10. If all the criteria mentioned above were fulfilled, the tracheostomy tube was replaced with a fenestrated uncuffed type of tube.

The proximal end of the tube was capped to allow breathing via normal air passages. The tracheostomy tube was capped for increasing periods in a stepwise manner. For the first 24 hours, capping was initially performed for at least 30 minutes every three hours during the day time. If there was no stridor, decrease in oxygen saturation, or signs of respiratory distress, the tracheostomy tube was capped during the daytime for 12 hours over the next 24 hours. This was followed by capping for 24 hours.
During this period, vital parameters including oxygen saturation were monitored closely by the nursing staff.

We excluded 15 patients (14.7\%) from the study as they did not meet the inclusion criteria. The reasons for exclusion were unstable mental status, ineffective cough reflex, requirement for frequent tracheal toileting, defective swallowing function with risk of aspiration, presence of subglottic stenosis due to granulation tissue, or because respiratory distress occurred shortly after capping of the tracheostomy tube. If a patient developed respiratory distress immediately after capping the tracheostomy tube, the first planned decannulation attempt was abandoned and the original tracheostomy tube was retained. Some of these patients underwent a second attempt at decannulation later when they improved clinically.

The remaining 87patients who fulfilled the decannulation criteria underwent tracheostomy tube removal. Decannulation was performed during early morning rounds. This allowed close monitoring of patients during the daytime when staff and equipment were available for airway management in case of failed decannulation. Patients were informed that the tracheostomy tube was going to be removed and warned of possible shortness of breath immediately after the weaning process. Decannulation was usually performed at the bedside with the patient in the supine position and the stoma was covered with a sterile gauze dressing. Patients were instructed to apply pressure with their fingers over the tracheal stoma while coughing or speaking to decrease air leakage. The tracheal stoma healed gradually over several days to weeks and closed without surgical intervention.

The sterile gauze dressing was replaced if it was soaked with secretions to enable rapid healing. Following decannulation, patients were continuously monitored for the next 48 hours for symptoms and signs of failure including dyspnea, stridor, tachypnea, tachycardia, and fall in oxygen saturation. Decannulation failure was defined as the requirement for re-establishing the airway by re-insertion of the tracheostomy tube within 48 hours of decannulation.

\section{RESULTS}

Table (1) showed decannulation criteria and Table 2 showed reasons for deferring decannulation on the first attempt. Table (3) showed that surgical tracheostomy was performed in the operating room for $58(67 \%)$ patients and the percutaneous dilatational technique was used in the intensive care unit for $29(33 \%)$ patients. Of the 87 patients analyzed, there were $42(48 \%)$ adult male and 37 $(43 \%)$ adult female patients, in addition to $8(9 \%)$ pediatric patients.

Among the female patients, 24(41\%) underwent surgical tracheostomy while $13(45 \%)$ underwent percutaneous dilatational tracheostomy. Among the male patients, 26 (45\%) underwent surgical 
tracheostomy while $16 \quad(55 \%)$ underwent percutaneous dilatational tracheostomy.All pediatric age group underwent surgical approach in the operating room.Decannulation was successful in 85/87 (97.7\%) patients. Successfully decannulated patients were discharged from our service within 2448 hours after decannulation with no need for further intervention or re-hospitalization. Failure of decannulation occurred in two cases $(2.3 \%)$.

On both occasions, the stoma was still evident, and a small-size tracheostomy tube was inserted using a tracheal dilator.

Table (1): Decannulation criteria

\begin{tabular}{|l|}
\hline 1. Conscious, oriented, and responsive to \\
commands \\
\hline $\begin{array}{l}\text { 2. The primary indication for tracheostomy no } \\
\text { longer exists }\end{array}$ \\
\hline $\begin{array}{l}\text { 3. All procedures requiring endotracheal } \\
\text { anesthesia were completed }\end{array}$ \\
\hline 4. Ventilator assistance no longer required \\
\hline $\begin{array}{l}\text { 5. Ability to mobilize secretions with the } \\
\text { requirement for tracheal suctioning of no more } \\
\text { than once per day }\end{array}$ \\
\hline $\begin{array}{l}\text { 6. } \text { Oxygen saturation was maintained at } 97 \% \text { or } \\
\text { more on room air }\end{array}$ \\
\hline 7. Intact, coordinated swallowing function \\
\hline 8. Intact protective cough reflex \\
\hline $\begin{array}{l}\text { 9. Airway patency confirmed by } \\
\text { laryngobronchoscopy }\end{array}$ \\
\hline $\begin{array}{l}\text { 10. Capping of the tracheostomy tube tolerated } \\
\text { for } 72 \text { hours }\end{array}$ \\
\hline
\end{tabular}

Table (2): Reasons for deferring decannulation on the first attempt

\begin{tabular}{|l|c|}
\hline \multicolumn{1}{|c|}{ Reasons } & No. of Patients \\
\hline $\begin{array}{l}\text { Altered mental status } \\
\text { (disorientation) }\end{array}$ & 2 \\
\hline Frequent suctioning & 5 \\
\hline $\begin{array}{l}\text { Uncoordinated swallowing } \\
\text { function }\end{array}$ & 4 \\
\hline $\begin{array}{l}\text { Tracheolaryngeal } \\
\text { pathology }\end{array}$ & 1 \\
\hline $\begin{array}{l}\text { Immediate capping } \\
\text { intolerance }\end{array}$ & 2 \\
\hline Ineffective coughing & 1 \\
\hline
\end{tabular}

Table (3): Patients' characteristics

\begin{tabular}{|l|l|c|}
\hline Characteristic & Mean age & $\begin{array}{c}\text { No. of } \\
\text { patients (\%) }\end{array}$ \\
\hline 1. Surgical tracheostomy \\
\hline Female & 65.0 years & $24(41 \%)$ \\
Male & 61.8 years & $26(45 \%)$ \\
Pediatric & 9.54 years & $8(14 \%)$ \\
\hline 2. Percutaneous dilatational tracheostomy \\
\hline Female & 59.5 years & $13(45 \%)$ \\
Male & 57.1 years & $16(55 \%)$ \\
\hline
\end{tabular}

\section{DISCUSSION}

Prolonged tracheostomy is usually associated with many complications as tracheal stenosis, aspiration pneumonia, and fistula or stricture formation ${ }^{(12)}$. Extremely serious bleeding has been recorded after development of fistula with the innominate artery (13). To avoid these serious complications decannulation process must be performed after resolution of the cause for the tube placement ${ }^{(13,14)}$.In accordant with the finding in our study many authors found that decannulation is a stepwise process and protocols may differ from one setting to another where physicians, otolaryngologists, respiratory therapists, and nurses have the maximum direct involvement in the decannulation process ${ }^{(14)}$.

In agreement with our detriments of decannulation authors found that the tracheostomy tube acts as a foreign body that can cause bronchorrhea or excessive cough and it impairs normal tracheal elevation while swallowing ${ }^{(11)}$. Significant adverse effects occur because the inspired gas bypasses the upper airway and enters the lumen of the tracheostomy directly ${ }^{(1,2)}$. The physiologic benefit of pursed-lip breathing is eliminated ${ }^{(3)}$. The vocal cords are bypassed, with no "laryngeal blast" to facilitate an effective cough and partial closing of the vocal cords induces subglottic stress called "physiological positive end-expiratory pressure"(4). Although tracheostomy is a common procedure, there is a lack of evidence as to when decannulation should be carried out ${ }^{(13,14)}$.

There is no universal agreement regarding the appropriate timing for tracheostomy weaning ${ }^{(1,7,13-15)}$. Various decannulation practices are described in the literature ${ }^{(12-14)}$. Different practical variations require the development of tracheostomy decannulation guidelines that may be applied in various clinical settings ${ }^{(12)}$. In this study, we followed an effective protocol, which was concordant with that of many reports and a success rate over $97 \%$ was achieved, which was comparable with the findings of other authors ${ }^{(11-16)}$.

The first patient with failed decannulation was 44-years-old, obese man, who had a severe chest infection with documented obstructive sleep apnea. He did not tolerate the first decannulation attempt as nocturnal oxygen desaturation was noted on the night of decannulation although he had tolerated capping of the tracheostomy tube. The second case of failed decannulation was a 62-years-old female patient who was diabetic, hypertensive, and had dyslipidemia. She was admitted to the intensive care unit following a cerebrovascular accident, complicated by nosocomial pneumonia. This patient fulfilled all decannulation criteria; however, after 2 hours of decannulation, she experienced significant shortness of breath. A small size tracheostomy tube was inserted; a large mucus 
plug was removed after vigorous suctioning of the trachea.

Decannulation was carried out successfully in both cases on the second attempt. There was no difference in successful decannulation rate between patients who underwent surgical or percutaneous dilatation tracheostomy.

\section{CONCLUSION}

The decision to perform elective decannulation of the tracheostomy tube can be challenging. However, we have demonstrated that decannulation based on objective criteria is effective in improving clinical judgment and weaning safety, with a low failure rate. We did not encounter any mortality or significant adverse events.

\section{REFERENCES}

1. Yu M (2010): Tracheostomy patients on the ward: multiple benefits from a multidisciplinary team?Crit Care, 14:109-11.

2. Mac Intyre NR, Cook DJ, Ely EW, Epstein SK, Fink JB, Heffner JE et al. (2001): Evidence-based guidelines for weaning and discontinuing ventilatory support: a collective task force facilitated by the American College of Chest Physicians; the American Association for Respiratory Care; and the American College of Critical Care Medicine. Chest, 120:375S95S.

3. Frutos-Vivar F, Esteban A, Apezteguía C, Anzueto A, Nightingale $P$, González M et al. (2005): Outcome of mechanically ventilated patients who require a tracheostomy. Crit Care Med., 33:290-8.

4. O'Connor H, White A (2010): Tracheostomy decannulation. Respir Care, 55:1076-81.
5. Pierson D (2005): Tracheostomy from A to $\mathrm{Z}$ : historical context and current challenges. Respir Care, 50:473-5.

6. Mc Clelland $R$ (1972): Tracheostomy; its management and alternatives. Proc R Soc Med., 65:401-4.

7. Golzari S, Khan Z, Ghabili K, Hosseinzadeh H, Soleimanpour H, Azarfarin $\mathrm{R}$ et al. (2013): Contributions of Medieval Islamic physicians to the history of tracheostomy. AnesthAnalg., 116:1123-32.

8. Goodall $\mathbf{E}$ (1934): On infectious diseases and epidemiology in the Hippocratic collection: (Section of the History of Medicine). Proc R Soc Med., 27:525-14.

9. Jackson C (1909):Tracheotomy. Laryngoscope, 19:285-90.

10. Lassen H (1953): A preliminary report on the 1952 epidemic of poliomyelitis in Copenhagen with special reference to the treatment of acute respiratory insufficiency. Lancet, 1:37-41.

11. Support S (2019): Decannulation . Available from: https://www.hopkinsmedicine.org/tracheostomy/livin g/decannulation.html

12. Epstein S (2005): Late complications of tracheostomy. Respir Care, 50:542-9.

13. Christopher K (2005): Tracheostomy decannulation. Respir Care, 50:538-41.

14. Maruvala S, Chandrashekhar R, Rajput R (2015): Tracheostomy decannulation: when and how? Research in Otolaryngology, 4:1-6.

15. Ranes J, Gordon S, Chen P, Fatica C, Hammel J, Gonzales J et al. (2006): Predictors of long-term mortality in patients with ventilator-associated pneumonia. Am J Med., 897: 3-9.

16. Cavaliere S, Bezzi M, Toninelli C, Foccoli P (2007): Management of post-intubation tracheal stenosis using the endoscopic approach. Monaldi Arch Chest Dis., 67:73-80. 